\title{
Insight into the Effects of Adipose Tissue Inflammation Factors on miR-378 Expression and the Underlying Mechanism
}

\author{
Xinye Jiang be Mei Xue $e^{\text {c,e }}$ Ziyi Fu Chenbo Jia Xirong Guo ${ }^{a}$ Lu Zhu Lulian Xu $^{\text {a }}$ \\ Lingxia Pang ${ }^{a}$ Meiyu $\mathrm{Xu}^{\mathrm{d}}$ Hongming $\mathrm{Qu}^{\mathrm{a}}$ \\ ${ }^{a}$ Affiliated Nanjing Maternity and Child Health Hospital, Nanjing Medical University, Nanjing, 'Wuxi \\ Maternal and Child Health Hospital, Wuxi, 'Taizhou City People's Hospital, Taizhou, 'Affiliated Hospital

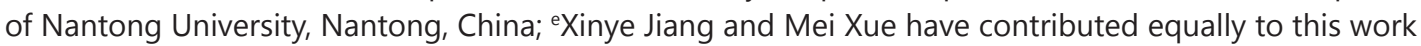

\section{Key Words}

Adipose $\bullet$ Inflammation factor $\bullet$ microRNA $\bullet$ Mechanism

\begin{abstract}
Background/Aims: Obesity and the related metabolic syndrome have emerged as major public health issues in modern society. miRNAs have been shown to play key roles in regulating obesity-related metabolic syndrome, and some miRNAs regulated by adiponectin were identified as novel targets for controlling adipose tissue inflammation. miR-378 is a candidate target that was shown to be involved in adipose differentiation, mitochondrial metabolism and systemic energy homeostasis. However, little is known about the regulatory mechanisms of miR-378 expression. To better understand the physiological role of miR-378 in obesity and metabolic syndrome, it is crucial that we understand the regulation of miR-378 gene expression in human adipocytes. Methods: In this study, we investigated the effects of adipokines and inflammatory cytokines on miR-378 expression using Real-time PCR and the potential regulatory mechanisms using luciferase reporter assays and electrophoretic mobility shift assay (EMSA). Results: We found that adipokines and cytokines upregulated miR-378 expression primarily through SREBP and C/EBP binding sites in the miR-378 promoter region. Conclusion: Our findings showed that adipokines induced miR-378 expression and revealed the most likely mechanism of adipokine-induced miR-378 dysregulation in human adipocytes. miRNAs have been shown to function in regulating obesity-related metabolic syndrome, and miR-378 may be a novel target for controlling adipose tissue inflammation. This study offers a theoretical basis for understanding systemic adipose tissue inflammation and may provide new strategies for clinical treatment.
\end{abstract}

Copyright (C) 2014 S. Karger AG, Basel

Meiyu Xu

and Hongming Qu
Nanjing Maternal and Child Health Medical Institute,

Affiliated Nanjing Maternal and Child Health Hospital, Nanjing Medical University، 123 Mochou Rd, Nanjing 210004 (China)

Tel. +86 25 52226159, Fax +86 25 84460507, E-Mail fybjqhm@163.com 


\section{Cellular Physiology Cell Physiol Biochem 2014;33:1778-1788 \begin{tabular}{l|l} 
and Biochemistry Published onlıne: Vlay 27, 2014 & $\begin{array}{l}\text { C) 2014 S. Karger AG, Basel } \\
\text { www.karger.com/cpb }\end{array}$ \\
\hline
\end{tabular} \\ Jiang et al.: The Mechanism of Regulating miR-378 Expression}

\section{Introduction}

Obesity and the related metabolic syndrome have emerged as major public health issues in modern society. Traditionally, obesity has been viewed as the accumulation of adipocyte tissue due to energy imbalance and adipocyte differentiation. It is now widely recognized that chronic low-grade inflammation [1,2] and oxidative stress [3], are involved in the initiation, propagation, and development of metabolic disorders [4]. Additionally, adipose tissue releases inflammatory cytokines and adipokines, such as leptin, resistin, tumor necrosis factor alpha (TNF- $\alpha$ ), interleukin-6 (IL-6), and adiponectin, which have been identified as major factors associated with obesity and metabolic syndrome [4-7].

MicroRNAs (miRNAs) are small noncoding RNAs (20 24 nt) that control gene expression by inducing target mRNA degradation or by blocking translation [8], and they are increasingly being recognized as viable therapeutic targets for a host of diseases. miRNAs have been proposed as therapeutic targets against cancer, HCV, HIV, and cardiovascular disease [9-12]. Recently, a growing body of evidence suggests that the deregulation of miRNAs is closely associated with obesity-related metabolic syndrome, including type 2 diabetes mellitus (T2DM) $[13,14]$. Furthermore, some miRNAs regulated by adiponectin were identified as novel targets for controlling adipose tissue inflammation $[15,16]$.

miR-378 is a candidate target that was shown to be involved in adipocyte gene expression, lipogenesis, the control of mitochondrial metabolism and systemic energy homeostasis [17, 18]. miR-378 is encoded by the peroxisome proliferator-activated receptor $\gamma$ coactivator $1 \beta(P G C-1 \beta)$ gene, which is a transcriptional coactivator that regulates metabolism and mitochondrial biogenesis and may counterbalance the metabolic actions of PGC-1 $\beta$ [19]. In 2010, Isabelle et al. reported that miR-378 stimulated the accumulation of triacylglycerol by specifically increasing the transcriptional activity of C/EBP $\alpha$ and C/EBP $\beta$ on adipocyte gene promoters [18]. In another study, Michele et al. reported that miR-378 was identified as an integral component of a regulatory circuit that functions under conditions of metabolic stress to control systemic energy homeostasis and the overall oxidative capacity of insulin target tissues [17]. However little is known about the regulatory mechanisms involved in miR-378 expression. To better understand the physiological role of miR-378 in obesity and metabolic syndrome, it is crucial that we understand the regulation of miR-378 gene expression in humans. In this study, we investigated the effects of adipokines and inflammatory cytokines on miR-378 expression and the potential regulatory mechanisms.

\section{Materials and Methods}

\section{Cell Culture and Treatment}

Human visceral preadipocytes (HPA-V; ScienCell Research Laboratories, USA) were maintained in preadipocyte medium (PAM; ScienCell Research Laboratories, USA), which contains $5 \%$ fetal bovine serum, $1 \%$ preadipocyte growth supplement, and $1 \%$ penicillin/streptomycin solution, at $37{ }^{\circ} \mathrm{C}$ in a humidified atmosphere of $5 \%$ carbon dioxide $\left(\mathrm{CO}_{2}\right)$. During the induced-differentiation phase, serum-free PAM (containing $50 \mathrm{nM}$ insulin, $100 \mathrm{nM}$ dexamethasone, $0.5 \mathrm{mM}$ 3-isobutyl-1-methylxanthine, and $100 \mathrm{lM}$ rosiglitazone) was added to confluent human preadipocytes (day 0), and the medium was replaced every 2 days for 4 days. Thereafter, the medium was replaced with serum-free PAM containing $50 \mathrm{nM}$ insulin and replaced every 2 days until lipid droplets had accumulated in the cells (day 15); fat accumulation was evaluated by staining 4\% paraformaldehyde-fixed cells with Oil Red O. Additionally, we collected the cells at different time points (day 0,1, 4, 7, 10,15). Differentiated adipocytes were used for experiments 15 days after the induction of differentiation, at which point over $80 \%$ of the cells showed the morphological and biochemical properties of adipocytes. After an overnight incubation in serum-free PAM, human adipocytes were treated with a final concentration of $30 \mathrm{ng} / \mathrm{ml} \mathrm{IL-6} \mathrm{[20]} \mathrm{(Sigma,} \mathrm{St.} \mathrm{Louis,} \mathrm{MO,} \mathrm{USA),} 10 \mathrm{ng} / \mathrm{ml}$ TNF- $\alpha$

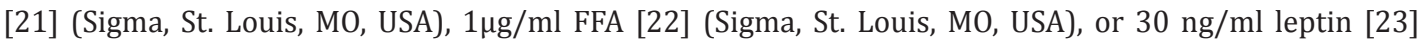
(Sigma, St. Louis, MO, USA). Human embryonic kidney 293T (HEK293T) cells were purchased from the American Type Culture Collection (Manassas, VA, USA) and maintained in Dulbecco's modified Eagle's 


\section{Cellular Physiology Cell Physiol Biochem 2014;33:1778-1788

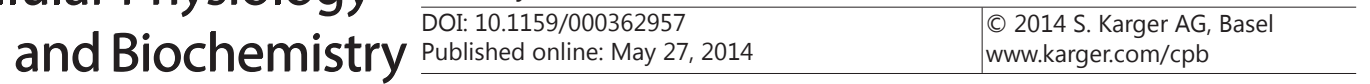 \\ Jiang et al.: The Mechanism of Regulating miR-378 Expression}

Table 1. The sequences of oligonucleotide primers used in promoter region PCR

\begin{tabular}{|c|c|c|}
\hline Promoter region & Product size (bp) & Forward and Reverse primers $\left(5^{\prime}-3^{\prime}\right)$ \\
\hline \multirow[t]{2}{*}{ Pro1 (-698bp to $-404 b p)$} & 295 & F: GGCAGGTCCCAGCCTCAGTA \\
\hline & & R: TTTGCCCTTTAGCCGCCCAG \\
\hline \multirow[t]{2}{*}{ Pro2 (-403bp to $-94 b p)$} & 310 & F: CGAGCCTAGCTAGCAGAAAT \\
\hline & & R: AGTGAGCGGCTTGTATGGGA \\
\hline \multirow[t]{2}{*}{ miR-378 (-698bp to $-94 b p)$} & 605 & F: GGCAGGTCCCAGCCTCAGTA \\
\hline & & R: AGTGAGCGGCTTGTATGGGA \\
\hline \multirow[t]{2}{*}{ miR-378/SREBP (-295bp to $-94 b p$ ) } & 202 & F: ATTTAACTGAATGACAGGCC \\
\hline & & R: AAAAAGCAAATGTCCCAGATT \\
\hline
\end{tabular}

medium (DMEM) supplemented with 10\% fetal bovine serum. HEK293T cells were also incubated at $37^{\circ} \mathrm{C}$ under a humidified atmosphere of $5 \% \mathrm{CO}_{2}$.

RNA Isolation and Quantitative Real-Time Reverse Transcriptase-Polymerase Chain Reaction (RT-PCR)

Total RNA from human adipocytes was extracted and purified using the TRIzol reagent (Invitrogen, USA) according to the manufacturer's protocol. The integrity of the RNA was assessed by agarose gel electrophoresis and measured by spectrophotometry at $260 \mathrm{~nm}$. To monitor the levels of miRNA, cDNA was synthesized from $200 \mathrm{ng}$ of total RNA using a TaqMan microRNA Reverse Transcriptase Kit (ABI, Foster City, CA, USA). qRT-PCR was performed using an Applied Biosystems 7500 Sequence Detection System (ABI 7500 SDS, USA) following the manufacturer's guidelines. Briefly, samples were incubated at $95{ }^{\circ} \mathrm{C}$ for $10 \mathrm{~min}$ for an initial denaturation, followed by 40 PCR cycles of incubation at $95{ }^{\circ} \mathrm{C}$ for $15 \mathrm{~s}$ and then $60{ }^{\circ} \mathrm{C}$ for 1 min. miRNA expression was normalized to snRNA U6 and miR-103 [16]. Primer identification numbers are 000567 for hsamiR- 378, 000439 for miR-103, and 001973 for snRU6 (ABI, Foster City, CA, USA). To detect mRNA expression levels, cDNA was synthesized from 500 ng of total RNA using a HighCapacity cDNA Reverse Transcriptase Kit (ABI, USA). The qRT-PCR primer sequences are as follows: $P G C$ $1 \beta$ forward, 5'-TGACTCCGAGCTCTTCCAG-3'; $P G C$-1 $\beta$ reverse, 5'-CGAAGCTGAGGTGCATGATA-3' [24]; GAPDH forward, 5'-CGGGTCGGGAGTGGGTAAT-3'; GAPDH reverse, 5'-AGTCGCCGTGCCTACCAT-3'. Data analysis was performed using the comparative CT method [25].

Plasmids and Promoter Reporter Constructs

We examined the upstream region of miR-378 using the UCSC genome browser (http://genome.ucsc. edu/). The two predicted promoter regions (pro1 and pro2) and the mutated form of the SREBP binding site of miR-378 (miR-378/SREBP: 5'-ATTTAACCACATGAC-3' mutated into 5'-ATTTAACTGAATGAC-3') were amplified by PCR from human genomic DNA using the primer sets shown in Table 1 . We reconstructed the pTA-Luc vector (Clontech, Mountain View, CA, USA) to form the vector pTB-Cherry. Then, the PCR products were subcloned into the pTB-Cherry vector using Nsi I/Xho I restriction sites (all constructs were confirmed by sequencing).

Luciferase Reporter Assays

HEK293T cells were grown to a density of 60-70\% confluence in 6-well plates and then were transiently transfected with $2 \mu \mathrm{g}$ of reporter plasmids per well with Lipofectamine 2000 (Invitrogen, USA) (using empty pTB-Cherry plasmid as a control). The upstream fragments induced the Cherry gene to express red fluorescence protein, and the promoter activity was determined by co-transfection of the pGL3-basic promoter reporter (250 ng/well) with $25 \mathrm{ng} /$ well pRL-TK (Renilla luciferase plasmid; Promega, Madison, Wisconsin) into HEK293T cells in 48-well plates. At $48 \mathrm{~h}$ post-transfection, the cells were harvested and cell lysates were prepared using a dual-luciferase reporter assay system according to the manufacturer's protocols (Promega) with a Luminometer (Turner Biosystems, USA). Firefly luciferase activity was normalized to Renilla luciferase activity. Furthermore, we detected the transcriptional activity of pro1, pro2, and miR-378 (PGL-3 basic as a control) with treatment of Rosiglitazone, TNF- $\alpha$, IL-6, or In5 separately.

\section{EMSA analysis}

The two predicted promoter regions (pro1 and pro2) and the mutated forms of SREBP or C/ EBP binding sites of miR-378 (SREBP: 5'-TTTATTTAACCACATGACAGGCCGAG-3', mutated into: 
Fig. 1. We collected cells at different time points (day $0,1,4,7,10,15$ ) and determined the expression levels of miR-378 (snRU6 was used as the internal control). The data showed that miR-378 was upregulated over time (Fig. 1A). To verify the expression pattern of miR-378, miR-103 was used for normalization, and the results were consistent with the data using snRU6 for normalization (Fig. 1B). ${ }^{*} \mathrm{p}<0.05$, ${ }^{* *} \mathrm{p}<0.01$. All experiments were performed independently in triplicate (minimum).

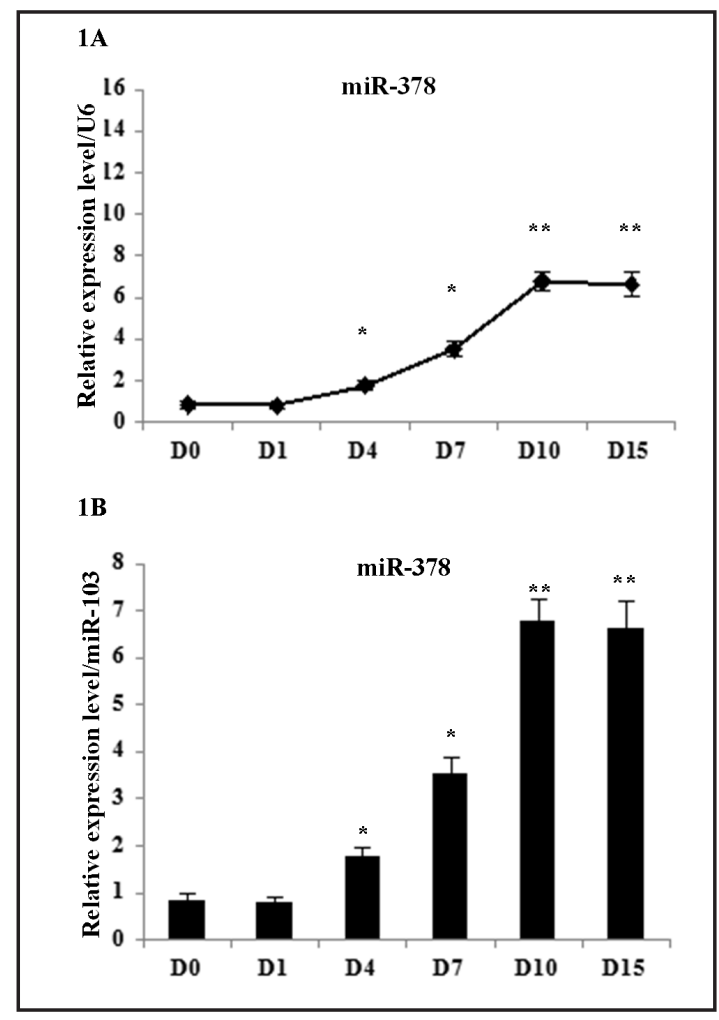

5'-TTTATTTAACCATTTTACAGGCCGAG-3', positive control: 5'-TTTGAAAATCACCCCATGCAAACTC-3'; C/ EBP: 5'-CCCAACTTGGGAAATGTAATT-3', mutated into 5'-CCCAACTTTTTTTTTGTAATT-3', positive control: 5 '-CCTTACCTTTTAGTCTTTCAACAAACT-3') were amplified by PCR from human genomic DNA using the primer sets shown in Table 1. The SREBP or C/EBP binding activity was detected using an EMSA Kit (Roche, Switzerland) according to the manufacturer's protocol. The preformed probes were set as the positive control. For the supershift assay, anti-SREBP or C/EBP antibody was added during preincubation. The visualized bands were analyzed using a BioSens Gel Imaging System (BIOTOP, China). For the competition experiment, 100-fold specific oligonucleotide competitor (unlabeled probe) was added to the binding mixture, and the reaction continued 10 min before the addition of the labeled probe. For mutant detection, 100 -fold mutated oligonucleotide was added to the binding mixture, and the reaction continued $10 \mathrm{~min}$ before the addition of the labeled probe.

\section{Statistical Analysis}

Data are presented as the mean \pm SEM. Statistical analysis was performed using one-way ANOVA. Differences between groups with $p<0.05$ were considered statistically significant. ${ }^{*} p<0.05$; ${ }^{* *} p<0.01$. All experiments were performed independently in triplicate (at minimum).

\section{Results}

miR-378 expression pattern during adipose differentiation

To determine the expression pattern of miR-378 during adipose differentiation in human adipocytes, we collected the cells at different time points (day $0,1,4,7,10,15$ ) and determined the expression levels of miR-378 using Real-time PCR (snRU6 was used as the internal control). The data showed that miR-378 was upregulated over time (Fig. 1A). To verify the expression pattern of miR-378, miR-103 was used for normalization, and the results were consistent with the data using snRU6 for normalization (Fig. 1B). 
Fig. 2. Mature adipocytes were treated with $30 \mathrm{ng} /$ ml IL-6, and miR378 expression level was examined at different time points $(0,4,8,24 \mathrm{~h})$ and normalized to snRU6 expression. We observed that miR-378 expression was significantly elevated with IL-6 (Fig. 2A), TNF- $\alpha$ (Fig. 2B), FFA (Fig. 2C) or leptin (Fig. 2D) treatment. ${ }^{*} \mathrm{p}<$ $0.05,{ }^{*} \mathrm{p}<0.01$. All experiments were performed independently in triplicate (at minimum).

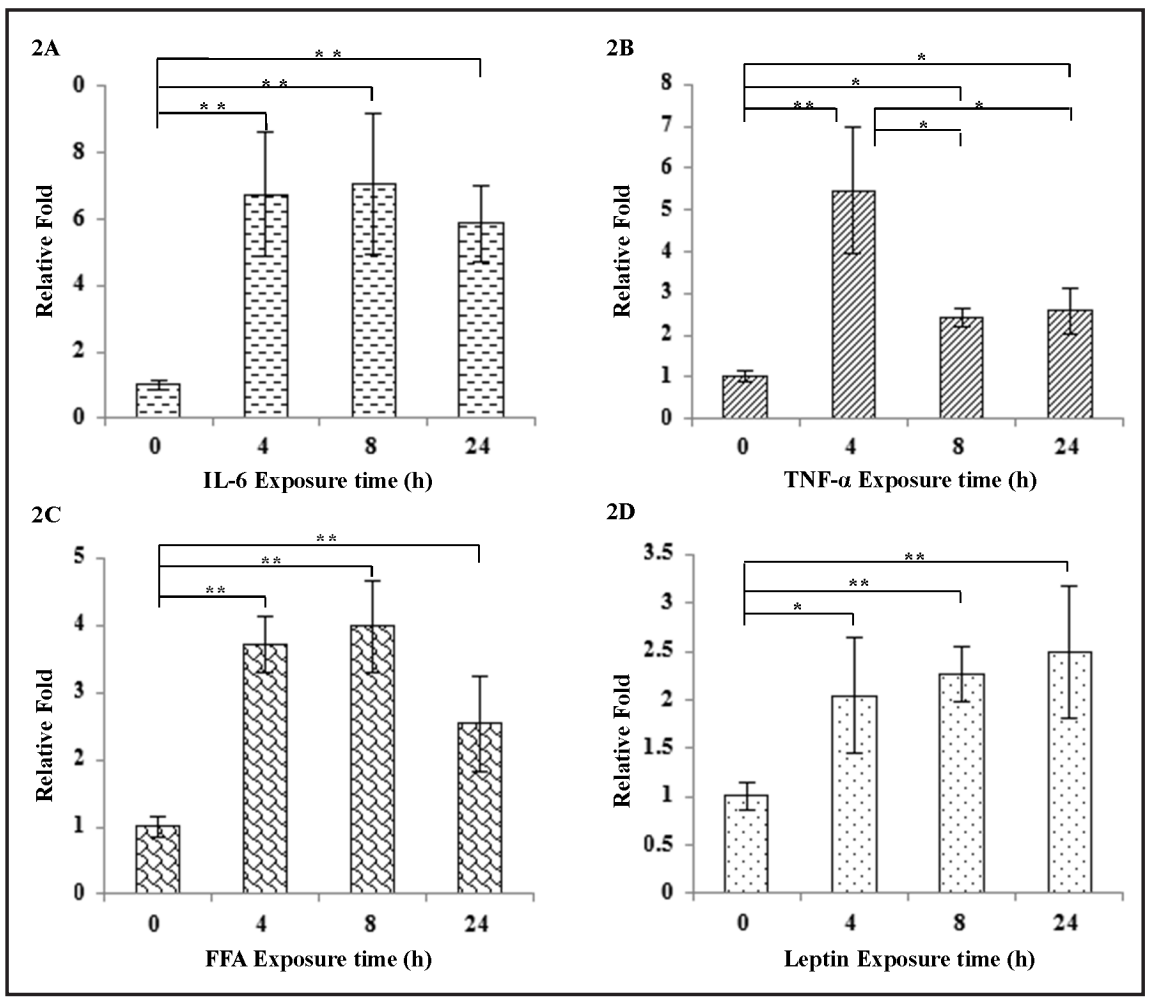

Fig. 3. We determined $P G C-1 \beta$ mRNA expression by real-time PCR. $P G C-1 \beta$ expression was evaluated from D4 to D10, and the expression peaked at D7 during adipogenesis. ${ }^{*} \mathrm{p}<0.05 ;{ }^{* *} \mathrm{p}<0.01$. All experiments were performed independently in triplicate (at minimum).

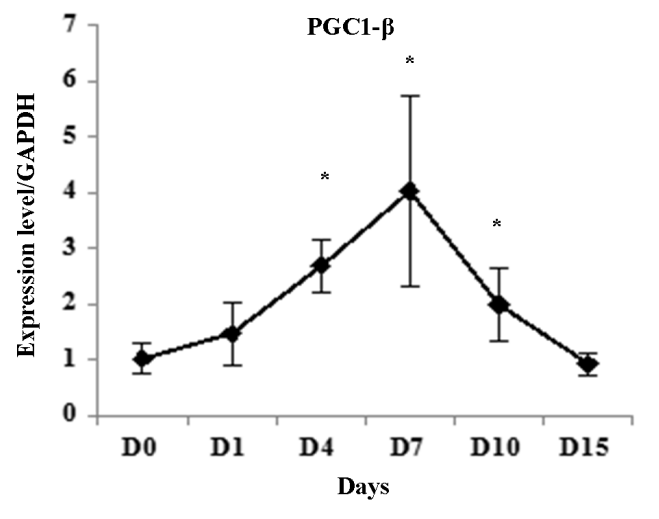

The effects of IL-6, TNF- $\alpha$, Leptin and FFA on miR-378 expression in Human Adipocytes

Mature adipocytes were treated with IL-6, and miR-378 expression was examined at different time points $(0,4,8,24 \mathrm{~h})$ and normalized to snRU6 expression. We observed that miR378 expression was significantly elevated $4 \mathrm{~h}$ following IL-6 exposure, with approximately 6.75-fold greater expression than the controls (Fig. 2A). miR-378 expression diminished at $24 \mathrm{~h}$ but, nevertheless, remained significantly upregulated compared to the control. To assess the effect of TNF- $\alpha$ treatment on miR-378 expression, mature adipocytes were incubated with TNF- $\alpha$. We observed that TNF- $\alpha$ significantly upregulated miR-378 (Fig. 2B). miR-378 expression was increased 5.47-fold at $4 \mathrm{~h}$, and miR-378 expression significantly diminished at $8 \mathrm{~h}$ and $24 \mathrm{~h}$ compared to $4 \mathrm{~h}$ but remained significantly upregulated compared to the control. Based on our results, FFA and leptin also had a significant effect on the expression of miR-378. When the cells were cultured with FFA, we detected a 3.7-fold upregulation of miR-378 expression at $4 \mathrm{~h}$ and a 2.5 -fold increase at $24 \mathrm{~h}$ (Fig. 2C). In addition, the level of miR-378 was increased by approximately 2-, 2.3-, and 2.5-fold at the different time points following treatment of the adipocytes with leptin (Fig. 2D). 
Fig. 4. We cloned two fragments from a 3-kb upstream region of hsa-miR-378 into the pTB-Cherry vector to generate pro1-pTB-Cherry and pro2pTB-Cherry plasmids. The results indicated that compared to PGL3-basic, pro1 had no effect on inducing gene expression, while pro2 (-403bp to $-94 \mathrm{bp}$ ) induced the expression of the downstream mCherry gene because full-length pro1 and pro2 (miR-378) showed increased promoter activity (Fig. 4). Then, a mutated form of the predicted SREBP binding site (miR-378/SREBP) was constructed in the pTB-Cherry vector, and the data showed that this mutant inhibited the promoter activity (Fig. 4). *p $<0.05$; ${ }^{* *}$ p $<$

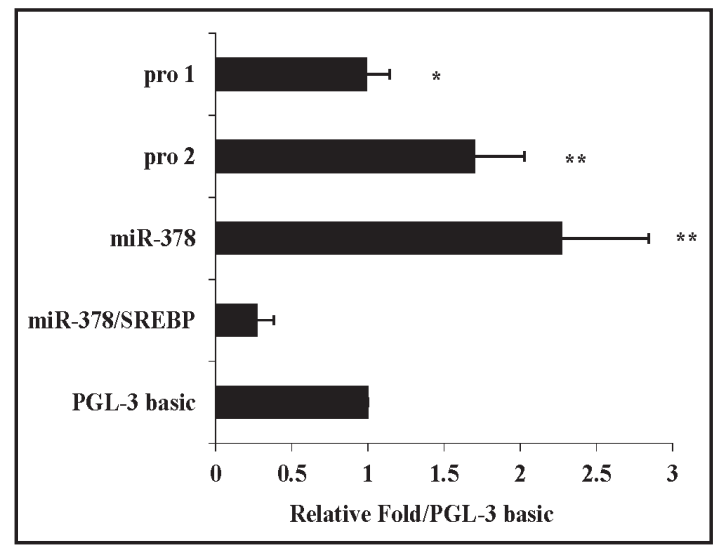
0.01 . All experiments were performed independently in triplicate (at minimum).

Fig. 5. We separately examined the activity of the constructs pro1, pro2, miR-378, miR-378/ SREBP, and PGL3-basic following Rosiglitazone, TNF- $\alpha$, IL-6, and In 5 treatment in HEK293T cells and found that pro1, pro 2 and miR-378 showed increased luciferase activity compared to PGL3-basic (Fig. 5). Interestingly, pro2 exhibited higher luciferase activity than pro 1 but lower than miR-378. *p $<$ $0.05 ;{ }^{* *} \mathrm{p}<0.01$. All experiments were performed independently in triplicate (at minimum).

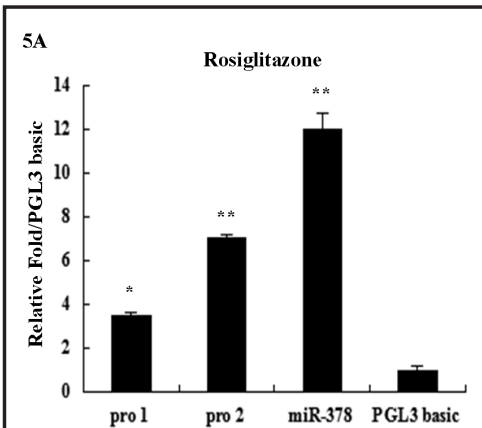

$5 \mathrm{C}$

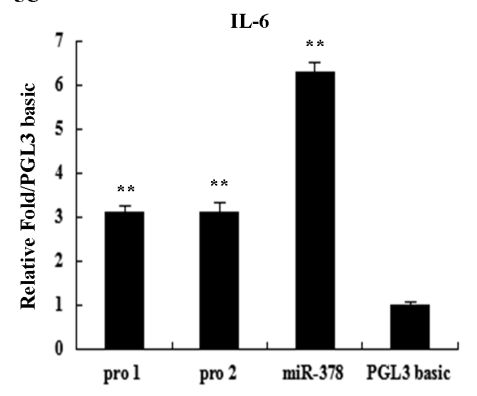

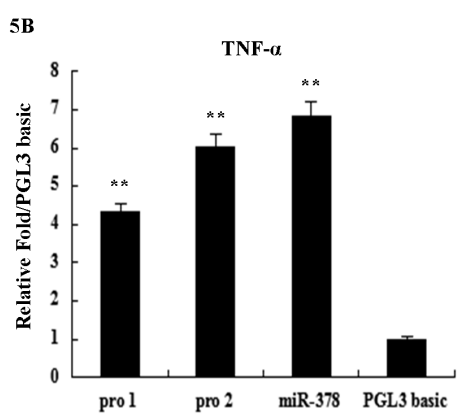

5D

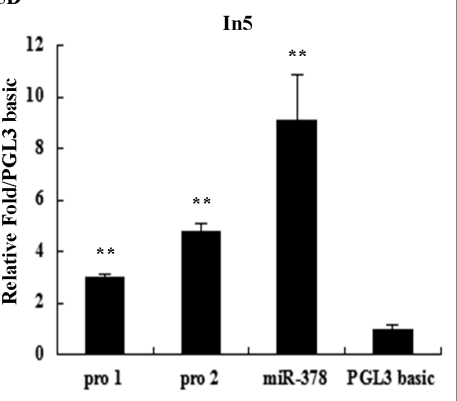

The Host Gene PGC-1 $\beta$ Expression is not Correlated with miR-378 in Human Adipocytes

miR-378 is an intronic miRNA encoded by the $P G C-1 \beta$ gene, and some intronic miRNAs are transcribed together with the host gene. Therefore, we determined $P G C-1 \beta$ mRNA expression using real-time PCR. Interestingly, $P G C-1 \beta$ expression was not correlated with the levels of miR-378 during adipogenesis (Fig. 3).

\section{Identification of Promoter Regions of miR-378}

To explore whether miR-378 has its own transcription unit, we subcloned two fragments from a 3-kb upstream region of hsa-miR-378 into the pTB-Cherry vector to generate pro1-pTB-Cherry and pro2-pTB-Cherry plasmids. We detected promoter activity in transfected HEK293T cells. The results indicate that compared to PGL3-basic, pro1 had no effect on inducing gene expression, while pro2 (-403 bp to $-94 \mathrm{bp}$ ) induced the expression of the downstream mCherry gene because full-length pro1 and pro2 (miR-378) showed increased promoter activity (Fig. 4). Then, a mutated form of the predicted SREBP binding site (miR-378/SREBP) was constructed in the pTB-Cherry vector, and the data showed that 
Fig. 6. To confirm the transcription factor binding sites, which are predicted to exist in the promoter region of miR-378, we performed an EMSA experiment. The data showed that Fig. 6A, Line1: positive control; line 2: sample; line 3: 100-fold unlabeled probe as a negative control; line 4: 100-fold labeled mutated-probe for the competition experiment; line 5: labeled probe and anti-SREBP antibody. Fig. 6B, Line1: positive control; line 2: sample; line 3: 100-fold unlabeled probe as a negative control; line 4: 100 -fold labeled mutated-probe for

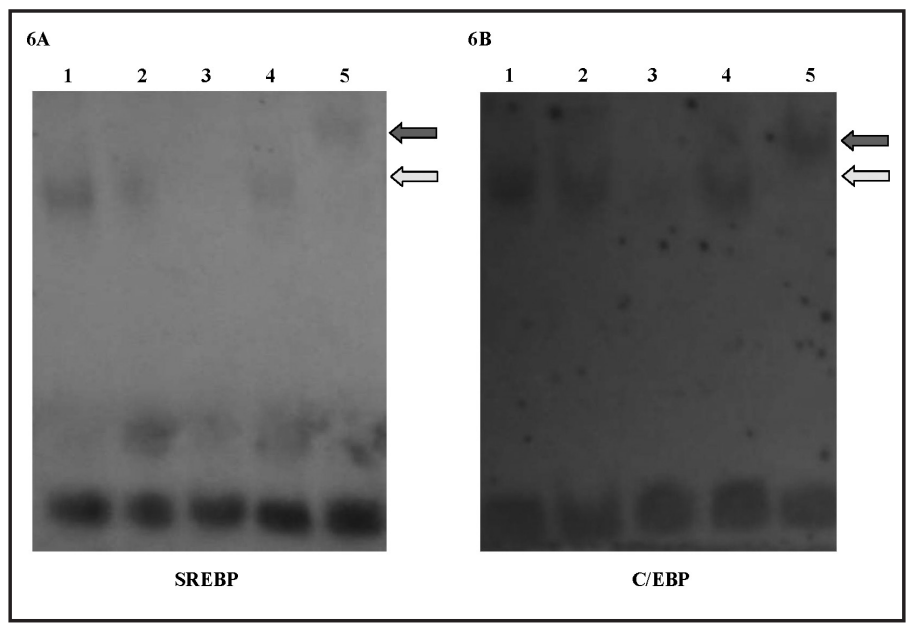
the competition experiment; line 5: labeled probe and anti-C/EBP antibody. The white arrow indicates the complex of the DIG-ddUTP-labeled oligonucleotide probe and SREBP or C/EBP. The black arrow indicates the oligonucleotide probe-labeled protein-SREBP or the protein-C/EBP antibody complex.

this mutant inhibited the promoter activity compared with the wild-type miR-378 (Fig. 4). We also examined the activity of these constructs by separate treatment with Rosiglitazone, TNF- $\alpha$, IL-6, and In5 in HEK293T cells and observed that pro1, pro 2 and miR-378 showed increased luciferase activity compared to PGL3-basic (Fig. 5). Interestingly, pro2 showed higher luciferase activity than pro 1 but lower than miR-378, which suggests that pro2 may be the major promoter of miR-378 and that there may be enhancers in pro1. Taken together, pro2 may be the major promoter of miR-378, and adipokines, such as Rosiglitazone, TNF- $\alpha$, IL-6, and In5, may induce miR-378 promoter activity.

\section{Confirmation of SREBP or C/EBP binding sites of miR-378}

Previously, we predicted that transcription factor binding sites existed in the promoter region of miR-378 and confirmed this prediction by performing an EMSA experiment. In this study, we constructed separate wild-type and mutated probes (labeled with biotin) of SREBP or C/EBP and preincubated them with the binding mixture for 10 min before the addition of the labeled probe. The data showed that both of the wild-type oligonucleotides conjugated to the target protein, while the mutated form did not bind to the target (Fig. 6). These results indicate that there are identified SREBP and C/EBP binding sites in the promoter region of miR-378.

\section{Discussion}

A growing body of evidence suggests that the levels of adipokines, such as IL- 6 , TNF- $\alpha$, leptin and FFA correlate with adipocyte differentiation. The dysregulation of these adipokines has been observed in obesity, type 2 diabetes, and cardiovascular diseases and an extended list of pathological changes to organs [26]. Recently, the dysregulation of miRNAs, including miR-378, was shown to closely associate with systemic energy homeostasis and the overall oxidative capacity of insulin target tissues [17]. However, little is known about the regulatory mechanisms involved in miR-378 expression. To better understand the physiological role of miR-378 in obesity and metabolic syndrome, it is crucial that we understand the regulation of miR-378 gene expression in humans. In this study, we investigated the effects of adipokines and inflammatory cytokines on miR-378 expression and the potential regulatory mechanisms. Interestingly, we observed that IL-6, TNF- $\alpha$, FFA, and leptin upregulated miR378 expression in human mature adipocytes. 
We first examined the expression pattern of miR-378 during adipocyte differentiation and found that miR-378 was upregulated over time compared with the endogenous control snRU6 (Fig. 1A). In addition, miR-103 has been shown to be the most stable miRNA transcript across all biological backgrounds in human adipose tissues derived from different origins [27]. Therefore, miR-103 was also used for normalization in this study, and the results were consistent with those using snRU6 (Fig. 1B).

Increased IL- 6 secretion has traditionally been thought to be induced by obesity, and IL-6 has been suggested as a novel link between obesity and metabolic syndrome [28]. Stouthard et al reported that increased glucose transport was observed in 3T3-L1 adipocytes following IL-6 treatment [29] and that IL-6 may lead to mitochondria dysfunction by inducing oxidative stress [30]. In addition, IL-6 has been shown to regulate adipocyte-related microRNA expression, such as the expression of miR-335 [16]. In this study, we explored the most likely effect of IL- 6 on miR-378 during adipocyte differentiation, and we observed that IL-6 induced miR-378 expression in human mature adipocytes. While Maarten et al found that miR-378 specifically increased the transcriptional activity of C/EBP on adipocyte gene promoters [18] and multiple studies have reported that C/EBP regulated IL-6 expression [31], it is possible that IL-6-induced miR-378 expression may create a feedback loop.

TNF- $\alpha$ is the first identified proinflammatory cytokine that provided a link between obesity, chronic inflammation, and insulin resistance [32]. Tili et al reported that TNF- $\alpha$ inhibited 3T3-L1 adipocyte differentiation by inducing miR-155 expression [33], and a several reports showed that TNF- $\alpha$ induced mitochondrial dysfunction in adipocytes, cardiac myocytes, and muscle [34-36]. Here, we treated human mature adipocytes with TNF- $\alpha$ and found that TNF- $\alpha$ increased miR-378 expression during adipocyte differentiation. Based on the known functions of miR-378 in adipocyte gene expression, namely the control of mitochondrial metabolism and systemic energy homeostasis $[17,18]$, TNF- $\alpha$ may induce mitochondrial dysfunction in adipocytes by increasing miR-378 expression. Further studies are needed to identify the possible link between miR-378 and TNF- $\alpha$-induced mitochondrial dysfunction.

Leptin is one of the most important adipocyte-derived hormones and helps regulate energy intake and metabolism [37]. It is well known that chronically elevated leptin levels are associated with obesity, inflammation-related diseases, and overeating. Recent studies have focused on the relationship between leptin and microRNAs in various diseases. Hamrick et al. demonstrated that leptin increased skeletal muscle mass by altering skeletal muscle miRNA expression profiles [38]. In another study, Zhu et al identified that leptin significantly increased miR-335 expression during adipocyte differentiation [16]. miR-378 expression levels were also elevated in human mature adipocytes after leptin exposure.

FFA is found in organisms and originates from the breakdown of triglycerides. FFAinduced insulin resistance may lead to type 2 diabetes and other cardiovascular risk factors in obesity. In 2008, Lovis et al demonstrated that the rise of miR-34a and miR-146 contributes to FFA-induced pancreatic $\beta$-cell dysfunction [39], and another study found that FFA inhibited the expression of tumor suppressor phosphatase and tensin homolog (PTEN) via miR-21 upregulation in hepatocytes [40]. In this study, we found that FFA increased miR378 expression during adipocyte differentiation, suggesting some underlying mechanisms involving the FFA-induced miR-378 upregulation.

Proinflammatory cytokines, such as TNF- $\alpha$, IL-1b, and IL-6, have been shown to play important roles in adipocyte biology [30]. Furthermore, there may be crosstalk among these factors during adipocyte differentiation. For example, elevated TNF- $\alpha$ levels may increase IL- 6 and MCP- 1 secretion in preadipocytes, and IL-6 exposure may inhibit the expression of adiponectin, resistin, glucose transporter type (GLUT)-4, and IRS-1 [41].

Our data show for the first time that these adipokines induce pronounced effects on miR-378 expression. To deduce the underlying mechanism for how adipokines regulate miR-378, we analyzed the genomic features of regions surrounding miR-378 and found that miR-378 was encoded within the gene $P G C-1 \beta$ (human chromosome $5 q 32$ ). PGC-1 $\beta$ is a transcriptional coactivator that regulates metabolism and mitochondrial biogenesis, and 
miR-378 may counterbalance the metabolic actions of PGC-1 [19]. Our results indicate that PGC-1 $\beta$ mRNA expression was clearly upregulated on day 7 during adipocyte differentiation, which was inconsistent with the miR-378 expression profile. Based on previous studies, some intronic microRNA transcription (such as miR-378) is initiated by promoters that are independent of their host genes $[19,42]$. We speculated that adipokines may regulate miR-378 transcription through an independent mechanism. Therefore, we subcloned and identified the potential promoter of miR-378 within the intron of the $P G C-1 \beta$ gene. Our results showed that the upstream region (-698bp to $-94 \mathrm{bp}$ ) of miR-378 contained its promoter and that there may be enhancers in this region (-403bp to $-94 \mathrm{bp})$. Interestingly, the promoter activity was upregulated by adipokines in HEK293T cells. The region of -403 bp to -94 bp analyzed by TFSEARCH (http://www.cbrc.jp/research/db/TFSEARCH.html) showed potential transcription factor binding sites (TFBS) for SREBP and C/EBP. To confirm this hypothesis, we performed an EMSA experiment. We constructed wild-type and mutated probes of the predicted binding sites for either SREBP or C/EBP, and we showed that both SREBP and C/EBP bound to the wild-type probes, indicating that these two transcriptional factors may regulate the expression of miR-378 by binding to sites within the miR-378 promoter. Our results show for the first time that adipokines and cytokines may induce miR378 expression primarily through SREBP and C/EBP binding sites in the miR-378 promoter region in human adipocytes. Further studies will be needed to confirm this mechanism.

In conclusion, our findings showed that adipokines induced miR-378 expression, demonstrating the most likely mechanism of adipokine-induced miR-378 dysregulation in human adipocytes. miRNAs have been shown to function in regulating obesity-related metabolic syndrome, and miR-378 may be a novel target for controlling adipose tissue inflammation. This study offers a theoretical basis for understanding systemic adipose tissue inflammation and may provide new strategies for clinical treatment.

\section{Acknowledgments}

This study was supported by grants from the National Key Basic Research Program of China (2013CB530604), the Key project of the National Natural Science Foundation of China (81330067), the National Natural Science Foundation of China $(81100618,81200642)$ and the Program for Innovative Research Teams of Jiangsu Province (LJ201108), and Nanjing Technological Development program (201104013).

\section{References}

1 Conen D, Rexrode KM, Creager MA, Ridker PM, Pradhan AD: Metabolic syndrome, inflammation, and risk of symptomatic peripheral artery disease in women: A prospective study. Circulation 2009;120:1041-1047.

2 Ridker PM, Buring JE, Cook NR, Rifai N: C-reactive protein, the metabolic syndrome, and risk of incident cardiovascular events: An 8-year follow-up of 14719 initially healthy american women. Circulation 2003;107:391-397.

3 Holvoet P, Kritchevsky SB, Tracy RP, Mertens A, Rubin SM, Butler J, Goodpaster B, Harris TB: The metabolic syndrome, circulating oxidized ldl, and risk of myocardial infarction in well-functioning elderly people in the health, aging, and body composition cohort. Diabetes 2004;53:1068-1073.

-4 Fonseca V, Desouza C, Asnani S, Jialal I: Nontraditional risk factors for cardiovascular disease in diabetes. Endocr Rev 2004;25:153-175.

5 Abate N, Sallam HS, Rizzo M, Nikolic D, Obradovic M, Bjelogrlic P, Isenovic ER: Resistin: An inflammatory cytokine. Role in cardiovascular diseases, diabetes and the metabolic syndrome. Curr Pharm Des 2013

6 Steppan CM, Bailey ST, Bhat S, Brown EJ, Banerjee RR, Wright CM, Patel HR, Ahima RS, Lazar MA: The hormone resistin links obesity to diabetes. Nature 2001;409:307-312. 
7 Wisse BE: The inflammatory syndrome: The role of adipose tissue cytokines in metabolic disorders linked to obesity. J Am Soc Nephrol 2004;15:2792-2800.

8 He L, Hannon GJ: Micrornas: Small rnas with a big role in gene regulation. Nat Rev Genet 2004;5:522-531.

-9 Houzet L, Klase Z, Yeung ML, Wu A, Le SY, Quinones M, Jeang KT: The extent of sequence complementarity correlates with the potency of cellular mirna-mediated restriction of hiv-1. Nucleic Acids Res 2012;40:11684-11696.

10 Janssen HL, Reesink HW, Lawitz EJ, Zeuzem S, Rodriguez-Torres M, Patel K, van der Meer AJ, Patick AK, Chen A, Zhou Y, Persson R, King BD, Kauppinen S, Levin AA, Hodges MR: Treatment of hcv infection by targeting microrna. N Engl J Med 2013;368:1685-1694.

11 Liu F, Yang T, Wang B, Zhang M, Gu N, Qiu J, Fan HQ, Zhang CM, Fei L, Pan XQ Guo M, Chen RH, Guo XR: Resistin induces insulin resistance, but does not affect glucose output in rat-derived hepatocytes. Acta Pharmacol Sin 2008;29:98-104.

12 Quiat D, Olson EN: Micrornas in cardiovascular disease: From pathogenesis to prevention and treatment. J Clin Invest 2013;123:11-18.

13 Heneghan HM, Miller N, Kerin MJ: Role of micrornas in obesity and the metabolic syndrome. Obes Rev 2010;11:354-361.

14 Hulsmans M, De Keyzer D, Holvoet P: Micrornas regulating oxidative stress and inflammation in relation to obesity and atherosclerosis. FASEB J 2011;25:2515-2527.

15 Ge Q, Gerard J, Noel L, Scroyen I, Brichard SM: Micrornas regulated by adiponectin as novel targets for controlling adipose tissue inflammation. Endocrinology 2012;153:5285-5296.

-16 Zhu L, Chen L, Shi CM, Xu GF, Xu LL, Zhu LL, Guo XR, Ni Y, Cui Y, Ji C: Mir-335, an adipogenesis-related microrna, is involved in adipose tissue inflammation. Cell Biochem Biophys 2013

17 Carrer M, Liu N, Grueter CE, Williams AH, Frisard MI, Hulver MW, Bassel-Duby R, Olson EN: Control of mitochondrial metabolism and systemic energy homeostasis by micrornas 378 and 378*. Proc Natl Acad Sci U S A 2012;109:15330-15335.

18 Gerin I, Bommer GT, McCoin CS, Sousa KM, Krishnan V, MacDougald OA: Roles for mirna-378/378* in adipocyte gene expression and lipogenesis. Am J Physiol Endocrinol Metab 2010;299:E198-206.

19 Eichner LJ, Perry MC, Dufour CR, Bertos N, Park M, St-Pierre J, Giguere V: Mir-378( *) mediates metabolic shift in breast cancer cells via the pgc-1beta/errgamma transcriptional pathway. Cell Metab 2010;12:352361.

20 Kralisch S, Klein J, Lossner U, Bluher M, Paschke R, Stumvoll M, Fasshauer M: Interleukin-6 is a negative regulator of visfatin gene expression in 3t3-11 adipocytes. Am J Physiol Endocrinol Metab 2005;289:E586590 .

-21 Wellen KE, Fucho R, Gregor MF, Furuhashi M, Morgan C, Lindstad T, Vaillancourt E, Gorgun CZ, Saatcioglu F, Hotamisligil GS: Coordinated regulation of nutrient and inflammatory responses by stamp2 is essential for metabolic homeostasis. Cell 2007;129:537-548.

-22 Nguyen MT, Satoh H, Favelyukis S, Babendure JL, Imamura T, Sbodio JI, Zalevsky J, Dahiyat BI, Chi NW, Olefsky JM: Jnk and tumor necrosis factor-alpha mediate free fatty acid-induced insulin resistance in 3t3-l1 adipocytes. J Biol Chem 2005;280:35361-35371.

-23 Wagoner B, Hausman DB, Harris RB: Direct and indirect effects of leptin on preadipocyte proliferation and differentiation. Am J Physiol Regul Integr Comp Physiol 2006;290:R1557-1564.

-24 Nanthirudjanar T, Furumoto H, Hirata T, Sugawara T: Oxidized eicosapentaenoic acids more potently reduce lxralpha-induced cellular triacylglycerol via suppression of srebp-1c, pgc-1beta and gpa than its intact form. Lipids Health Dis 2013;12:73.

25 Schmittgen TD, Livak KJ: Analyzing real-time pcr data by the comparative c(t) method. Nat Protoc 2008;3:1101-1108.

26 Guilherme A, Virbasius JV, Puri V, Czech MP: Adipocyte dysfunctions linking obesity to insulin resistance and type 2 diabetes. Nat Rev Mol Cell Biol 2008;9:367-377.

27 Neville MJ, Collins JM, Gloyn AL, McCarthy MI, Karpe F: Comprehensive human adipose tissue mrna and microrna endogenous control selection for quantitative real-time-pcr normalization. Obesity (Silver Spring) 2011;19:888-892.

28 Younossi ZM, Jarrar M, Nugent C, Randhawa M, Afendy M, Stepanova M, Rafiq N, Goodman Z, Chandhoke V, Baranova A: A novel diagnostic biomarker panel for obesity-related nonalcoholic steatohepatitis (nash). Obes Surg 2008;18:1430-1437. 


\section{Cellular Physiology Cell Physiol Biochem 2014;33:1778-1788 \begin{tabular}{l|l|l} 
DOI: 10.1159/000362957 & $\begin{array}{l}\text { O 2014 S. Karger AG, Basel } \\
\text { www.karger.com/cpb }\end{array}$
\end{tabular}

29 Stouthard JM, Oude Elferink RP, Sauerwein HP: Interleukin-6 enhances glucose transport in 3t3-11 adipocytes. Biochem Biophys Res Commun 1996;220:241-245.

30 Hotamisligil GS: Inflammation and metabolic disorders. Nature 2006;444:860-867.

31 Rego D, Kumar A, Nilchi L, Wright K, Huang S, Kozlowski M: Il-6 production is positively regulated by two distinct src homology domain 2-containing tyrosine phosphatase-1 (shp-1)-dependent ccaat/ enhancer-binding protein beta and nf-kappab pathways and an shp-1-independent nf-kappab pathway in lipopolysaccharide-stimulated bone marrow-derived macrophages. J Immunol 2011;186:5443-5456.

32 Tzanavari T, Giannogonas P, Karalis KP: Tnf-alpha and obesity. Curr Dir Autoimmun 2010;11:145-156.

-33 Tili E, Michaille JJ, Cimino A, Costinean S, Dumitru CD, Adair B, Fabbri M, Alder H, Liu CG, Calin GA, Croce CM: Modulation of mir-155 and mir-125b levels following lipopolysaccharide/tnf-alpha stimulation and their possible roles in regulating the response to endotoxin shock. J Immunol 2007;179:5082-5089.

-34 Chen XH, Zhao YP, Xue M, Ji CB, Gao CL, Zhu JG, Qin DN, Kou CZ, Qin XH, Tong ML, Guo XR: Tnf-alpha induces mitochondrial dysfunction in 3t3-11 adipocytes. Mol Cell Endocrinol 2010;328:63-69.

-35 Mariappan N, Soorappan RN, Haque M, Sriramula S, Francis J: TNF-alpha-induced mitochondrial oxidative stress and cardiac dysfunction: Restoration by superoxide dismutase mimetic tempol. Am J Physiol Heart Circ Physiol 2007;293:H2726-2737.

-36 Valerio A, Cardile A, Cozzi V, Bracale R, Tedesco L, Pisconti A, Palomba L, Cantoni O, Clementi E, Moncada S, Carruba MO, Nisoli E: TNF-alpha downregulates eNOS expression and mitochondrial biogenesis in fat and muscle of obese rodents. J Clin Invest 2006;116:2791-2798.

-37 Scott MM, Williams KW, Rossi J, Lee CE, Elmquist JK: Leptin receptor expression in hindbrain glp-1 neurons regulates food intake and energy balance in mice. J Clin Invest 2011;121:2413-2421.

-38 Hamrick MW, Herberg S, Arounleut P, He HZ, Shiver A, Qi RQ, Zhou L, Isales CM, Mi QS: The adipokine leptin increases skeletal muscle mass and significantly alters skeletal muscle mirna expression profile in aged mice. Biochem Biophys Res Commun 2010;400:379-383.

-39 Lovis P, Roggli E, Laybutt DR, Gattesco S, Yang JY, Widmann C, Abderrahmani A, Regazzi R: Alterations in microrna expression contribute to fatty acid-induced pancreatic beta-cell dysfunction. Diabetes 2008;57:2728-2736.

40 Vinciguerra M, Sgroi A, Veyrat-Durebex C, Rubbia-Brandt L, Buhler LH, Foti M: Unsaturated fatty acids inhibit the expression of tumor suppressor phosphatase and tensin homolog (pten) via microrna-21 upregulation in hepatocytes. Hepatology 2009;49:1176-1184.

41 Chung S, Lapoint K, Martinez K, Kennedy A, Boysen Sandberg M, McIntosh MK: Preadipocytes mediate lipopolysaccharide-induced inflammation and insulin resistance in primary cultures of newly differentiated human adipocytes. Endocrinology 2006;147:5340-5351.

42 Liu M, Roth A, Yu M, Morris R, Bersani F, Rivera MN, Lu J, Shioda T, Vasudevan S, Ramaswamy S, Maheswaran S, Diederichs S, Haber DA: The IGF2 intronic mir-483 selectively enhances transcription from IGF2 fetal promoters and enhances tumorigenesis. Genes Dev 2013;27:2543-2548. 